\title{
Commentary
}

Neurocysticercosis (NC), the larval stage of taeniasis/ cysticercosis, is a well-recognized public health problem worldwide, not only in low and middle-income countries, but also in the high income ones, due to the globalization and migration.

The paper of Kasundra et al. ${ }^{[1]}$ highlights that many aspects of NC are still unknown. Extraneural cysticercosis is frequent in Africa and Asia, but it is extremely rare in Latin America. Although the genetic differences in the Taenia solium specimens from each continent could participate in this difference, host genetic factors may also be involved. ${ }^{[2]}$ Due to technological advances, one major improvement in NC knowledge has been the identification of cysts by means of imaging (magnetic resonance imaging [MRI] and computed tomography [CT]), allowing diagnosis of $\mathrm{NC}$ and evaluation of treatment properly. It is crucial to keep in mind that from the epidemiological, pathophysiological, immunological and clinical point of views, the two locations of the parasite in the central nervous system (CNS), parenchymal and extraparenchymal, are two entirely different entities. The clinical manifestations, mainly seizures, due to parenchymal location are usually of good prognosis and are sometimes transitory in time. On the contrary, clinical presentation of the extraparenchymal location is life threatening and may lead to permanent sequelae such as hydrocephalus and cognitive decline. ${ }^{[3]}$

The inflammatory response also depends on the location of the parasite. When located in the parenchyma, inflammation will be confined around the parasite. In these cases, the cerebrospinal fluid (CSF) is generally normal. When parasites are located in the subarachnoid space or in the ventricular system, inflammation will be mainly seen in the CSF with the presence of lymphocytic pleocytosis, mild elevation of protein, and hypoglycorrhachia. ${ }^{[3,4]}$ This is associated with an intense inflammatory reaction, fibrosis, and progressive thickening of the leptomeninges at the base of the brain. ${ }^{[5]}$ There is an obstruction of the CSF circulation, resulting in hydrocephalus and progressive intracranial hypertension. Inflammation of the meninges, cranial nerve involvement, chiasmatic syndrome, and cerebral infarcts secondary to vasculitis may develop ${ }^{[3]}$ Mortality related to hydrocephalus is high $(50 \%)$, and most patients die within 2 years after CSF shunting. ${ }^{[5]}$ When cysticerci are located inside the ventricular system, acute intracranial hypertension as a result of hydrocephalus may occur. Cysts in the subarachnoid space may lodge in the Sylvian fissure or basal cisterns and grow to a large size (racemose form), also causing intracranial hypertension.

The clinical utility of serologic assays for NC is limited. Detection of specific antibodies in sera using enzyme-linked immunosorbent assay (ELISA) and enzyme-linked immunoelectrotransfer blot (EITB) assay do not differentiate between viable and degenerated parasites, and are not specific to CNS localization. ${ }^{[4]}$ These assays are useful for identification of individuals who have had contact with the parasite at some time, but are not specific to active infection in the CNS. ${ }^{[4]}$ However, detection of specific antigens in sera by ELISA in patients with parasites located in the subarachnoid space or the ventricular system is a specific sign of parasite viability and may be used to evaluate treatment response. ${ }^{[6]}$ 
$\mathrm{CT}$ and MRI are useful in diagnosis of NC and in the evaluation the evolutionary stage of the parasite, as well as number and localization of parenchymal lesions. Extraparenchymal NC is more difficult to detect by imaging because the attenuation and signal intensity of the cyst's content is similar to that of CSF and the cystic wall is usually not detected, and the cysts frequently lack a scolex and are generally not enhanced after contrast administration. ${ }^{[3,4]}$ New MRI techniques such as fluid-attenuated inversion recovery (FLAIR) and fast imaging employing steady-state acquisition (FIESTA) sequences permit better detection of the parasites. ${ }^{[7]}$

Antihelminthic treatment of NC appears to only be effective in eradicating active parenchymal cysts in about one third of patients. ${ }^{[4]}$ So far, there are no evidence-based studies that have showed efficacy of antihelminthic treatment for extraparenchymal NC. In a double-blind placebo-controlled trial, disappearance of extraparenchymal cysts with albendazole was not significantly different than placebo. ${ }^{[8]}$ Neurosurgical intervention should be considered for hydrocephalus requiring ventriculoperitoneal shunt or intraventricular localization of a cyst. ${ }^{[4]}$

Diagnosis of NC is currently based on epidemiological, clinical, imaging and serological tests. Unfortunately, all these criteria present limitations and gold standard for the diagnosis of NC is only pathology ${ }^{[4]}$ This favors misdiagnosis that can lead to inappropriate treatment. As parenchymal and extraparenchymal NC are two different entities, it is not possible to apply a single set of diagnostic criteria to them. Diagnostic criteria are needed that differentiate between both locations.

Del Brutto et al.$^{[9]}$ proposed diagnostic criteria for NC. Despite being published more than 10 years ago, they have not been validated. These diagnostic criteria may be useful to identify patients with mainly parenchymal forms of NC, but have limited use for patients with extraparenchymal NC. ${ }^{[10]}$ One of the main problems is its complexity, but also its subjectivity, since there are no operational definitions for most concepts used.

Properly designed studies are needed to understand the natural history of the disease and the true distribution of clinical manifestations. There is also an urgent need to develop new treatment options and alternative consensus diagnosis criteria for NC, particularly for extraparenchymal NC. Last but not the least, prevention and measures to eradicate taeniasis/cisticercosis in endemic countries require improving environmental health conditions at the population level. Eradication relies on social, economic and political factors, highlighting the need for national governments to make of this a public health priority.

\section{Arturo Carpio}

Department of Neurology School of Medicine, University of Cuenca, Ecuador, G.H. Sergievsky Center, Columbia University, NY, USA

Address for correspondence: Prof. Arturo Carpio, School of Medicine, Av, 12 de Abril s/n, El Paraiso, Cuenca, Ecuador. E-mail: arturo.carpio@ucuenca.edu.ec

\section{References}

1. Kasundra GM, Bhargava AN, Bhushan B, Khichar S, Sood I. Disseminated neurocysticercosis presenting as isolated acute monocular painless vision loss. J Neurosci Rural Pract 2014;5:S89-92.

2. Fleury A, Escobar A, Fragoso G, Sciutto E, Larralde C. Clinical heterogeneity of human neurocysticercosis results from complex interactions among parasite, host and environmental factors. Trans R Soc Trop Med Hyg 2010;104:243-50.

3. Fleury A, Carrillo-Mezo R, Flisser A, Sciutto E, Corona T. Subarachnoid basal neurocysticercosis: A focus on the most severe form of the disease. Expert Rev Anti Infect Ther 2011;9:123-33.

4. Carpio A, Fleury A, Hauser WA. Neurocysticercosis: Five new things. Neurol Clin Pract 2013;3:118-25.

5. Sotelo J, Marin C. Hydrocephalus secondary to cysticercotic arachnoiditis. A long-term follow-up review of 92 cases. J Neurosurg 1987;66:686-9.

6. Michelet L, Fleury A, Sciutto E, Kendjo E, Fragoso G, Paris L, et al. Human neurocysticercosis: Comparison of different diagnostic tests in cerebrospinal fluid. J Clin Microbiol 2011;49:195-200.

7. Neyaz Z, Patwari SS, Paliwal VK. Role of FIESTA and SWAN sequences in diagnosis of intraventricular neurocysticercosis. Neurol India 2012;60:646-7.

8. Carpio A, Kelvin EA, Bagiella E, Leslie D, Leon P, Andrews H, et al.; Ecuadorian Neurocysticercosis Group. Effects of albendazole treatment on neurocysticercosis: A randomized controlled trial. J Neurol Neurosurg Psychiatry 2008;79:1050-5.

9. Del Brutto OH, Rajshekhar V, White AC Jr, Tsang VC, Nash TE, Takayanagui OM, et al. Proposed diagnostic criteria for neurocysticercosis. Neurology 2001;57:177-83.

10. Machado Ldos R. Neurocysticercosis: Still some unanswered questions. Arq Neuropsiquiatr 2014;72:329-30.

\begin{tabular}{|l|l|}
\hline \multicolumn{2}{|c|}{ Access this article online } \\
\hline Quick Response Code: & \\
\hline & Website: \\
\hline
\end{tabular}

